\title{
OPEN Pirfenidone modifies hepatic miRNAs expression in a model of MAFLD/NASH
}

\author{
Rebeca Escutia-Gutiérrez ${ }^{1}$, J. Samael Rodríguez-Sanabriaa ${ }^{1}$, C. Alejandra Monraz-Méndez ${ }^{1}$, \\ Jesús García-Bañuelos ${ }^{1}$, Arturo Santos-García ${ }^{2}$, Ana Sandoval-Rodríguez ${ }^{1,3 凶}$ \& \\ Juan Armendáriz-Borunda ${ }^{1,2,3 凶}$
}

miRNAs are involved in the development of metabolic associated fatty liver disease (MAFLD) and nonalcoholic steatohepatitis (NASH). We aimed to evaluate modifications by prolonged-release pirfenidone (PR-PFD) on key hepatic miRNAs expression in a MAFLD/NASH model. First, male C57BL/6J mice were randomly assigned into groups and fed with conventional diet (CVD) or high fat and carbohydrate diet (HFD) for 16 weeks. At the end of the eighth week, HFD mice were divided in two and only one half was treated with $300 \mathrm{mg} / \mathrm{kg} /$ day of PR-PFD mixed with food. Hepatic expression of miRNAs and target genes that participate in inflammation and lipid metabolism was determined by qRT-PCR and transcriptome by microarrays. Increased hepatic expression of miR-21a-5p, miR-34a-5p, miR-122-5p and miR-103-3p in MAFLD/NASH animals was reduced with PR-PFD. Transcriptome analysis showed that 52 genes involved in lipid and collagen biosynthesis and inflammatory response were downregulated in PR-PFD group. The expression of $I l 1 b, T n f a, I l 6, T g f b 1$, Col1a1, and Srebf1 were decreased in PR-PFD treated animals. MAFLD/NASH animals compared to CVD group showed modifications in gene metabolic pathways implicated in lipid metabolic process, inflammatory response and insulin resistance; PR-PFD reversed these modifications.

Metabolic associated fatty liver disease (MAFLD), previously known as non-alcoholic fatty liver disease is currently the most common cause of chronic liver disease worldwide ${ }^{1,2}$. MAFLD is characterized by hepatic steatosis accompanied by one of three features: overweight or obesity, type 2 diabetes mellitus (T2DM), or either lean or normal weight with evidence of metabolic dysregulation ${ }^{3}$. MAFLD prevalence is rising due to the global obesity epidemic and high prevalence of type II diabetes mellitus ${ }^{4,5}$. It is estimated that up to a third of the world population will present the disease in a nearby future ${ }^{6,7}$. Population studies have estimated a prevalence of $17-22 \%$ in asymptomatic patients ${ }^{8,9}$. The progressive form of MALFD condition; nonalcoholic steatohepatitis (NASH), is defined by liver cell damage, lobular inflammatory infiltrate, and a variable degree of fibrosis ${ }^{10}$. Several microRNAs (miRNAs) are involved in MAFLD progression and NASH pathology. miRNAs are highly conserved non-coding RNAs that epigenetically regulate gene expression at post-transcriptional level. miRNAs involved in lipid synthesis, catabolism of fatty acids and glucose and inflammation, are known to be dysregulated in MAFLD/NASH ${ }^{11,12}$; including miR-122-5p, miR-34a-5p, miR-21a-5p and miR-103-3p ${ }^{13-16}$. miR-122-5p is the most expressed miRNA in adult human liver (approximately $70 \%$ of total miRNAs) and its key role in total serum cholesterol regulation and liver lipids metabolism has been clearly demonstrated ${ }^{17,18}$. miR-34a-5p is involved in lipid metabolism, apoptosis, cell cycle control, and studies in mice have shown how overexpression of miR-34a-5p increases the level of liver triglycerides ${ }^{19,20}$. Likewise, it has been demonstrated that miR-103-3p regulates insulin sensitivity and glucose homeostasis ${ }^{21,22}$; while miR-21a-5p may be involved at different stages of MAFLD progression, in buildup of lipids, appearance of steatosis in hepatocytes and/or inflammation at early stages and fibrosis at a later stage of the disease ${ }^{23,24}$.

Several drugs are currently being investigated for the treatment of NASH, notwithstanding, there are no current FDA approved therapies for treating NASH. Given the vast complexity of NASH where lipid metabolism, insulin resistance, oxidative stress, inflammation and fibrosis pathways are involved, a drug with multiple

\footnotetext{
${ }^{1}$ Department of Molecular Biology and Genomics, Institute for Molecular Biology in Medicine and Gene Therapy, Health Sciences University Center, University of Guadalajara, Guadalajara, Jalisco, Mexico. ${ }^{2}$ Tecnologico de Monterrey, School of Medicine and Health Sciences, Zapopan, Jalisco, Mexico. ${ }^{3}$ Institute for Molecular Biology and Gene Therapy, CUCS, University of Guadalajara, 950 Sierra Mojada, 44340 Guadalajara, Mexico. ${ }^{\square}$ email: anasol44@hotmail.com; armdbo@gmail.com
} 
biological targets ought to be required to stimulate beneficial effects, if any ${ }^{25,26}$. Pirfenidone (PFD) is a pyridonederived drug with extensive antifibrotic, anti-inflammatory and antioxidant effects which modulates a number of cytokines, i.e., Tgfb1, Il1, Il4, Il6, Il8, Il13, IFNG and Tnfa, among others. Furthermore, PFD reduces expression of intracellular adhesion molecule 1 (Icam-1) and improves anti-inflammatory cytokines expression, such as $I l 10^{27,28}$. In this work we used prolonged-release pirfenidone (PR-PFD) with an improved bioavailability compared to standard pirfenidone and a half-life of $\sim 8 \mathrm{~h}^{29}$. Thus, the aim of this study was to evaluate modifications by PR-PFD on key hepatic miRNAs expression in a MAFLD/NASH model.

\section{Methods}

Drug. Prolonged-release pirfenidone (PR-PFD) was donated by Cell Pharma S.A. de C.V. (Cuernavaca, Mexico). Chemical reagents used were reactive grade.

Animals. CUCS Research Committee at the University of Guadalajara approved this study (protocol number: CI-00518). The study was carried out in compliance with the ARRIVE guidelines. All mouse assays were in compliance with the Animal Research Reporting in Vivo Experiments guidelines and accomplished according to guidelines for the care and use of laboratory animals published by the US National Institutes of Health (NIH, publication No. 85-23, revised 1996). Four-week-old male C57BL/6J mice $(n=15)$, weighing 18-22 g, were obtained from Cinvestav Animal Facility (Mexico City). After a week of acclimatization, animals were divided into groups; fed with conventional diet (CVD) with 18\% lipids (2018S.15-Envigo) and plain water or fed with high fat/high carbohydrate diet (HFD) with 60\% fat nutriment (TD.06414-Envigo) and 42gr sugar/L in drinking water (55\% fructose and $45 \%$ sucrose) for 16 weeks. From ninth week to the end of the protocol, a subgroup of five HFD mice were treated with prolonged release pirfenidone (PR-PFD) mixed with food ( $300 \mathrm{mg} / \mathrm{kg} / \mathrm{day}$ ). Mice were maintained with 12-h light/dark cycles, ad libitum food and water; and constant temperature and humidity. Animals were weighed weekly to control weight gain.

Sacrifice, clinical and biochemical parameters. Animals were sacrificed after $4 \mathrm{~h}$ of fasting using tiletamine/zolazepam (15 mg/kg/bw, Zoletil 50, Virbac) at the end of the 16th week of model induction. Liver and whole blood were collected. The weight of epididymal fat and liver were determined. Blood was centrifuged to obtain serum where lipid profile (triglycerides, total cholesterol and VLDL) and liver enzymes (AST, ALT) were determined using VITROS DT60II dry chemistry analyzer (Johnson \& Johnson).

Insulin tolerance test and determination of glucose levels and HOMA. Fasting blood glucose was measured weekly during the study in mouse-tail vein using a clinical glucometer. The Insulin Tolerance Test (ITT) was determined at eighth and sixteenth week. After $4 \mathrm{~h}$-fasting, $0.025 \mathrm{U} /$ mouse of short-acting human recombinant insulin was intraperitoneally administrated and glucose levels were measured at $0,30,60$ and 90 min after insulin injection. The area under the curve was calculated using the trapezoidal method ${ }^{30}$. Insulin was measured in serum by multiplex detection immunoassay. Insulin resistance (IR) was calculated using the homeostasis model assessment (HOMA)-IR index with the following formula [fasting serum glucose $(\mathrm{mg} / \mathrm{dL})$ $\times$ fasting serum insulin $(\mu \mathrm{IU} / \mathrm{mL}) / 405]^{31}$.

Histological analysis of liver. Samples from the three main liver lobes were fixed in $4 \%$ paraformaldehyde (0.1 M PBS, pH 7.4). Paraffin embedded tissues were cut into $5 \mu \mathrm{m}$ sections. Hematoxylin-eosin, Masson's trichrome and Sirius red staining were performed. Masson's and H\&E were analyzed by two independent pathologists to quantify inflammation, microvesicular steatosis and macrovesicular steatosis. Microvesicular steatosis was defined by the presence of numerous small lipid vacuoles that do not displace the nucleus; while macrovesicular steatosis was defined by a large lipid vacuole that displaces the nucleus. In addition, periportal and centrilobulillar fibrosis were examined by scoring fibrotic bridges. To evaluate hepatic stellate cells activation, immunoreactivity against alpha-smooth muscle actin (Cell Signaling Technology Inc., Beverly MA, USA) and anti-GFAP (Glial Fibrillary Acidic Protein) (Biocare Medical, Pacheco, CA, USA) was performed using a 1:50 and 1:100 ab dilution respectively. To evaluate inflammatory infiltration in the liver immunoreactivity against CD68 (Biocare Medical, Pacheco, CA, USA) was carried out using a 1:100 ab dilution.

Collagen, alpha-smooth muscle actin, GFAP and CD68 staining were evaluated using Image-Pro software in 30 photographs at $20 \times$ microscopic field magnification (Media Cybernetics, Inc., Bethesda MD, USA).

RT-qPCR. RNA isolation from liver was performed according to Chomczynski and Sacchi modified method $^{32}$. RNA quantity and quality were determined with NanoDrop equipment (Thermo Scientific, USA). $2 \mu \mathrm{g}$ of total RNA were used for retrotranscription with $240 \mathrm{ng}$ Oligo dT, $0.5 \mathrm{mM}$ dNTPs mix, $10 \mathrm{mM}$ DTT, 2 U of RNAse inhibitor and $200 \mathrm{U}$ M-MLV (Invitrogen, Carlsbad, CA). qPCR reactions were performed on the LightCycler 96 Instrument (Roche Molecular Systems, Pleasanton, CA, USA). All data were run in triplicate, normalized using GAPDH as housekeeping gene and data analysis was performed using the $2^{-\Delta c t} \operatorname{method}^{33}$. Information about the probes used is shown in Supplemental Table 1.

miRNAs extraction and expression. Representative liver tissue was stored in RNAlater stabilization solution (Ambion, Austin, TX, USA) at $-80^{\circ} \mathrm{C}$. miRNAs were extracted using the miRVana miRNA isolation kit (Thermo Fisher Scientific, Waltham, MA, USA), according to manufacturer's instructions. The integrity and amount of the RNA fraction highly enriched in sRNA species $\leq 200$ nt was determined using a Nanodrop ND-2000 spectrophotometer (Thermo Fisher Scientific, Waltham, MA, USA). RNA purity was assessed from 


\begin{tabular}{|c|c|c|c|}
\hline \multirow[b]{2}{*}{ Parameter } & \multicolumn{3}{|l|}{ Group } \\
\hline & CVD & HFD & PFD \\
\hline \multicolumn{4}{|l|}{ Body weight (g) } \\
\hline Initial & $17.38 \pm 0.31$ & $20.60 \pm 0.67$ & $19.30 \pm 0.46$ \\
\hline Final & $27.98 \pm 0.47$ & $49.25^{\mathrm{a}} \pm 2.36^{\star * *}$ & $40.33^{\mathrm{b}} \pm 2.82^{*}$ \\
\hline Weight gain $(g)$ & $10.60 \pm 0.51$ & $29.54^{\mathrm{a}} \pm 1.84^{* * *}$ & $20.83^{\mathrm{b}} \pm 2.97^{*}$ \\
\hline Epididimal fat pad weight (g) & $0.67 \pm 0.11$ & $2.60 \pm 0.33$ & $1.79^{\mathrm{b}} \pm 0.16^{*}$ \\
\hline Relative liver weight $(\mathrm{g})$ & $4.85 \pm 0.34$ & $4.7 \pm 0.30$ & $4.7 \pm 0.30$ \\
\hline Macrovesicular steatosis (\%) & - & $19.2 \pm 1.1$ & $8.0^{\mathrm{b}} \pm 1.8^{* * * *}$ \\
\hline Microvesicular steatosis (\%) & - & $71.2 \pm 1.7$ & $67.7 \pm 2.3$ \\
\hline Inflammatory nodules number/total area & - & $14.8 \pm 2.1$ & $6.60^{\mathrm{b}} \pm 0.96^{* * *}$ \\
\hline Fibrotic bridges number/total area & - & $4.6 \pm 1.9$ & $0.6^{\mathrm{b}} \pm 0.2^{*}$ \\
\hline Periportal fibrosis (\%) & - & $72.5 \pm 7.3$ & $34.7^{\mathrm{b}} \pm 3.8^{\star *}$ \\
\hline Pericental fibrosis (\%) & - & $34.2 \pm 3.3$ & $20.5^{\mathrm{b}} \pm 3.1^{\star}$ \\
\hline AST (U/L) & $116.7 \pm 6.7$ & $225.0^{\mathrm{a}} \pm 8.6^{* * * *}$ & $140.0^{\mathrm{b}} \pm 19.2^{* * * *}$ \\
\hline $\operatorname{ALT}(\mathrm{U} / \mathrm{L})$ & $36.0 \pm 2.3$ & $91.0^{\mathrm{a}} \pm 1.7^{* * * *}$ & $42.0^{\mathrm{b}} \pm 2.8^{* * *}$ \\
\hline Total colesterol (mg/dL) & $78.0 \pm 6.3$ & $153.0^{\mathrm{a}} \pm 7.7^{\star}$ & $120.0^{\mathrm{b}} \pm 6.9^{*}$ \\
\hline Triglycerides $(\mathrm{mg} / \mathrm{dL})$ & $128.0 \pm 8.0$ & $212.0^{\mathrm{a}} \pm 12.7^{\star * * *}$ & $127.0^{\mathrm{b}} \pm 10.9^{* * * *}$ \\
\hline $\mathrm{VLDL}(\mathrm{mg} / \mathrm{dL})$ & $30.0 \pm 1.7$ & $34.0 \pm 2.0$ & $25.0^{\mathrm{b}} \pm 2.3^{*}$ \\
\hline Serum glucose (mg/dL) & $110.7 \pm 6.93$ & $207.0^{\mathrm{a}} \pm 14.21^{\star *}$ & $177.3 \pm 5.48$ \\
\hline AUC & $9.65 \pm 0.62$ & $18.09 \pm 1.69$ & $11.73^{\mathrm{b}} \pm 0.35^{*}$ \\
\hline Insulin $(\mu \mathrm{IU} / \mathrm{mL})$ & $2490 \pm 321.6$ & $4326^{\mathrm{a}} \pm 446.3^{*}$ & $4710^{\mathrm{a}} \pm 719.4^{*}$ \\
\hline HOMA-IR index ${ }^{c}$ & $1.0 \pm 0.06$ & $3.25^{\mathrm{a}} \pm 0.36^{\star *}$ & $2.7 \pm 0.54$ \\
\hline
\end{tabular}

Table 1. Clinical, histological and biochemical parameters in animal groups. Data are expressed as mean \pm SEM. CVD conventional diet, HFD high fat and high carbohydrate diet, $P F D$ high fat diet + prolonged release pirfenidone. $V L D L$ very low-density lipoproteins. ${ }^{a}$ Significance difference versus CVD group $\left({ }^{*} \mathrm{p}<0.05\right.$, $\left.{ }_{* * * *} \mathrm{p}<0.0001\right)$. ${ }^{\mathrm{b}}$ Significance difference versus HFD group $\left({ }^{*} \mathrm{p}<0.05,{ }^{* *} \mathrm{p}<0.01,{ }^{* * *} \mathrm{p}<0.001,{ }^{* * * *} \mathrm{p}<0.0001\right)$. ${ }^{\mathrm{c}}$ Fasting serum glucose $(\mathrm{mg} / \mathrm{dL}) \times$ fasting serum insulin $(\mu \mathrm{IU} / \mathrm{mL}) / 405$.

the ratio of A260/A280; where 1.8-2.1 is expected ${ }^{34}$. Ten ng of sRNAs were used to performed retrotranscription using the Advanced miRNA cDNA synthesis kit (Thermo Fisher Scientific, Waltham, MA, USA), following the manufacturer's instructions. Reverse transcription products were diluted to $50 \mu \mathrm{L}$, and $2.5 \mu \mathrm{L}$ of the diluted sample were used for qPCR reactions, with a total volume of $10 \mu \mathrm{L} / \mathrm{Rx}$ and $1 \mathrm{X}$ Thermo specific TaqMan probes were used for qPCR for mmu-miR-34a-5p, mmu-miR-122-5p, mmu-miR-21a-5p and mmu-miR-103-3p, in a LigthCycler 96 equipment (Roche Molecular Systems, Pleasanton, CA, USA). qPCR was performed as follows: 1 cycle at $95^{\circ} \mathrm{C}$ for $20 \mathrm{~s}, 40-60$ cycles $95^{\circ} \mathrm{C}$ for $1 \mathrm{~s}$ followed by $60^{\circ} \mathrm{C}$ for $20 \mathrm{~s}$. Probes catalog number are shown in Supplemental Table 1. Gene expression was normalized against mmu-miR-16-5p. Analysis was performed using the $2^{-\triangle \mathrm{CT}}$ method $^{35}$.

Microarrays. Total RNA was extracted from $20 \mathrm{mg}$ of liver tissue using Trizol reagent (Invitrogen, Carlsbad, CA, USA). Abundance and ratio between 28 and 18S rRNA were monitored both by agarose gel electrophoresis and NanoDrop ND-2000 spectrophotometer lectures (Thermo Fisher Scientific, Waltham, MA, USA). Total RNA pooled from 5 animals per group was used throughout the experiment. Reverse transcription and RNA sample labeling was made according to Microarray Facility UNAM protocol (http://microarrays.ifc.unam. $\mathrm{mx})^{36}$. Double-channel microarrays for Mus musculus genome were used and 22,000 genes were hybridized. Image quantification was analyzed with genArise Microarray Analysis tool version 2.0 with adjusted p values of $<0.05$ and $\mathrm{Z}$ score values of $>1.5$ and $<1.5$ were considered to be significant. To provide functional interpretation of enlisted genes, Database for Annotation, Visualization and Integrated Discovery (DAVID) v6.8 was employed to visualized the cell pathways and processes involved in non-alcoholic steatohepatitis and modifications induced by PR-PFD ${ }^{37}$.

Statistical analysis. Values are expressed as mean \pm SD, or as mean \pm SEM. Groups were compared with one-way ANOVA followed by Tukey test for parametric data and Kruskal-Wallis or U de Mann-Whitney for non-parametric data. Values of $\mathrm{p}<0.05$ were considered statistically significant. Analysis was run with GraphPad Prism version 8 .

\section{Results}

Pirfenidone improves clinical and biochemical parameters in MAFLD/NASH animal model. All animals gradually increased their weight during the experimental period. As seen in Table 1 after being fed a high-fat diet for 16 weeks, HFD mice showed a 2.8-fold increase in body weight; while PFD group gain a 1.96fold increase in weigh compared to CVD group. CVD fed animals showed $27.98 \mathrm{~g} \pm 0.47$ of body weight. HFD 
mice treated with PR-PFD, diminished body weight $18.11 \%$ against HFD group (40.33 g \pm 2.82 vs $49.25 \mathrm{~g} \pm 2.36$; $\mathrm{p}<0.05)$. These data indicated that PFD-treated animals even when fed with HFD, ended with lesser weight gain and final body weight. No statistical differences in liver weight or liver/animal weight ratio were observed in any experimental group (Table 1). Epididymal fat pad showed a significant decrease in PFD group compared to animals in HFD group ( $1.79 \mathrm{~g} \pm 0.16$ vs $2.60 \mathrm{~g} \pm 0.33 ; \mathrm{p}<0.05)$ (Table 1$)$. Also, PFD group displayed a significant decrease in AST and ALT serum levels $(\mathrm{p}<0.0001)$ versus HFD group (Table 1$)$. Triglycerides, cholesterol and VLDL serum levels were higher in HFD group mice against PFD group $(\mathrm{p}<0.0001)$ (Table 1$)$. Animals treated with pirfenidone had a slight decrease in serum glucose compared to HFD group. However, mice receiving PFD treatment showed increased insulin sensitivity (AUC determinations), which was statistically significant at the end of week 16, $(\mathrm{p}<0.05)$. Insulin levels were increased in HFD animals compared to CVD group $(4326 \pm 446.3$ vs $2490 \pm 321.6 ; \mathrm{p}<0.05$ ) (Table 1). Insulin showed no statistical differences between HFD and Pirfenidone group. Insulin resistance was increased in HFD group, as assessed by HOMA-IR, yet an increase in this hormone sensitivity was observed in mice treated with PFD.

Pirfenidone modifies MAFLD/NASH-involved miRNAs expression while improving liver inflammation and steatosis. Several miRNAs involved in MAFLD/NASH development where explored using RT-qPCR in liver tissue. Also, in order to correlate changes in miRNAs expression, some of their target genes were selected on miRbase and RT-qPCR was performed and/or were searched in the microarray database of PFD vs HFD chip. Liver levels of miR-21a-5p, a key regulator of lipid metabolism and hepatic fibrogenesis, were significantly higher $(27.28 \pm 5.27)$ in HFD mice compared to CVD mice $(4.8 \pm 0.85 ; \mathrm{p}<0.01)$. HFD mice receiving PFD treatment exhibited a significant lower expression $(12.52 \pm 1.15 ; \mathrm{p}<0.05)$ (Fig. 1A). Srebf1 and $T g f b 1$ mRNA hepatic expression were determined using PCR. Srebfl mRNA levels were lower in mice administered with PFD $(24.82 \pm 2.62 ; \mathrm{p}<0.05)$ versus HFD group $(52.94 \pm 13.51)$, while $T g f b 1$ also revealed statistical negative difference $(17.00 \pm 2.67$ vs $36.09 \pm 1.53 ; \mathrm{p}<0.0001)$ (Fig. 1A). Additionally, data on microarray analysis showed upregulation in miR-21a-5p-target genes like Fabp1 (2.24) and Ppara (0.16); while Hbp1 and Myd88 showed downregulation ( -0.20 and -0.15 , respectively).

miR-122-5p levels are elevated under lipid buildup conditions, with quantities correlating with NASH scoring and severity. As shown in Fig. 1B, our results revealed that miR-122-5p was upregulated in hepatic tissue of HFD fed mice, and concurrent treatment with PFD decreased its liver expression $(1559 \pm 296.50$ vs $730.80 \pm 85.52$; $\mathrm{p}<0.01$ ). Target genes for miR-122-5p like Fasn, Mttp, Sirt1 and Ppard showed in microarray profile - 0.71, $-0.38,1.72$ and 1.16 expression values compared to HFD animals. While qPCR expression of Fasn was found to decrease $(3.08 \pm 0.15 ; \mathrm{p}<0.05)$ in PFD group against HFD group $(4.70 \pm 0.54)$. By qPCR, $M t t p$ gene expression showed non-statistical difference between groups.

miR-34a-5p is usually expressed in the liver, modulating oxidative stress and lipid metabolism. Figure 1C shows its increased expression in HFD group compared to PFD administered animals ( $5060 \pm 2844$ vs $1264 \pm 149.0 ; p=0.12)$. Noteworthy, PFD group levels are similar to those obtained in CVD group $(1208 \pm 260.4)$. miR-103-3p has been shown to regulate insulin sensitivity and glucose homeostasis and is highly expressed in the liver of MAFLD patients. Data in Fig. 1D for miR-103-3p indicated an upregulation in HFD animals $(150 \pm 85.08)$ and a notable decrease in its expression after PFD treatment $(42.14 \pm 7.19 ; \mathrm{p}=0.25)$. In microarray assays, its target gene Fgf5 was found downregulated ( 1.82$)$; while Cpt1 $a$ was found upregulated (0.84) in PFD group. Concurrent with this finding, Cpt1a is upregulated in animals treated with PFD $(1.630 \pm 0.3047, \mathrm{p}<0.05)$ compared to the high fat diet group $(0.792 \pm 0.05)$.

MAFLD/NASH-related parameters are decreased in liver tissue of animals after pirfenidone administration. Histological analysis of liver of animals fed with HFD group showed significant tissue damage with steatosis, imminent fibrosis, and inflammatory changes predominantly in the periportal area with neutrophils and mononuclear cells. After prolonged-release pirfenidone treatment; as shown in H\&E staining, a significant reduction in inflammation foci was achieved $(\mathrm{p}<0.001)$ (Table 1, Fig. 2). To correlate this data, macrophage recruitment in the liver was evaluated using CD68 marker (Fig. 3A). Quantification of CD68 positive area was increased in HFD animals compared to PFD group. $(6.29 \pm 1.03 v s .1 .57 \pm 0.34, \mathrm{p}<0.0001)$. In particular, macrovesicular steatosis reached substantial reduction in PFD group compared to HFD mice group (Table 1, $\mathrm{p}<0.0001$ ); while microvesicular steatosis decrease in PFD group did not reached statistical significance (Fig. 2, Table 1). An imminent fibrosis development was observed, especially in periportal and pericentral areas. Fibrosis assessment was higher in HFD group; while histological improvement was observed in PFD animals $(\mathrm{p}<0.01$ and $\mathrm{p}<0.05$ for periportal and pericentral zones, respectively) (Fig. 2 Sirius red and Masson staining, Table 1). Likewise, a significant decrease in the number of fibrotic bridges was observed after administration of PFD versus HFD group ( $\mathrm{p}<0.05$; Fig. 2 Masson staining, Table 1). To corroborate these facts, Sirius red collagen staining revealed lesser reactivity in PDF treated animals compared to non-treated HFD group ( $<<0.05$, Fig. $2 B$ ).

Hepatic stellate cells activation in a MAFLD/NASH model was reversed in PFD treated animals. In order to monitor changes in HSC activation, immunohistochemistry for alpha-SMA was realized. As indicated in Fig. 2C, images were analyzed and percentage of area with immunoreactivity was calculated. HFD group showed a $2.28 \pm 0.36 \%$ predominantly in the periportal area; whereas PFD group only had a $0.275 \pm 0.055 \%(\mathrm{p}<0.01)$ indicating reduction of HSC activation in treated animals. This data also correlates with lesser fibrosis and collagen staining in this group. To evaluate if pirfenidone decrease $\alpha$-SMA positive cells is due to reversal into the HSC quiescent phenotype, we performed GFAP immunohistochemistry (Fig. 3B). GFAP positive area was increased in PFD animals compared to HFD group. ( $3.96 \pm 0.45$ vs. $1.13 \pm 0.2, \mathrm{p}<0.0001)$. 
A

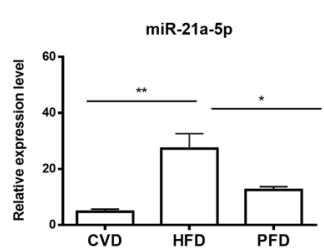

B

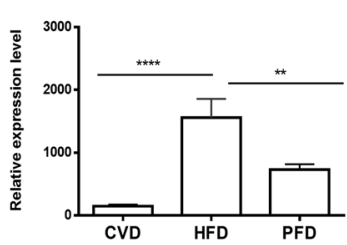

C

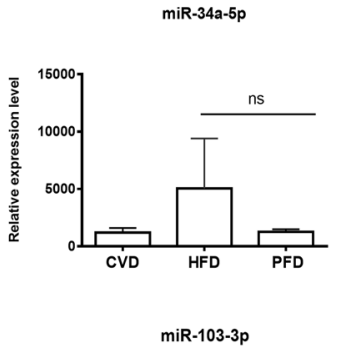

D

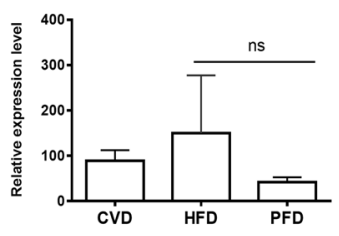

Srebf1

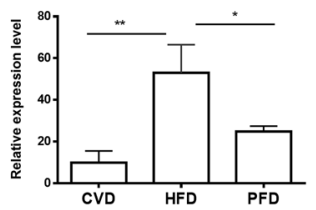

Fasn

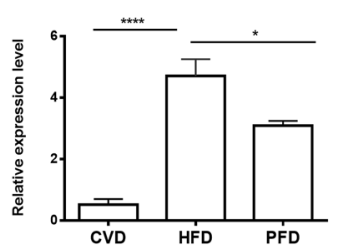

Target genes of miR-34a-5p

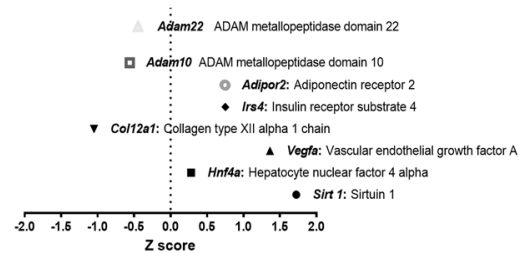

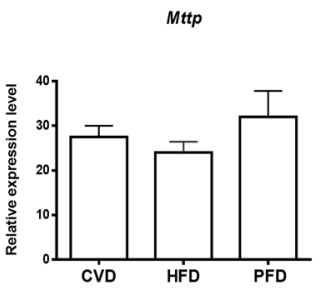

$T g f b 1$

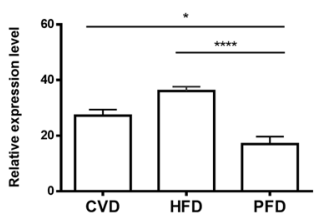

Mttp
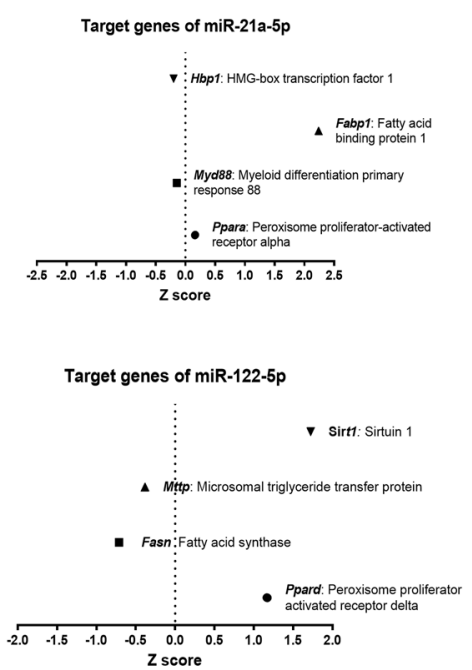

$\begin{array}{rrrrrrrrr}-2.0 & -1.5 & -1.0 & -0.5 & 0.0 & 0.5 & 1.0 & 1.5 & 2.0\end{array}$

Figure 1. Hepatic expression of selected miRNAs involved in the pathogenesis of non-alcoholic steatohepatitis and their target genes. (A) miR-21a 5p, Srebf1 and $T g f b 1$ gene expression by qPCR. Final panel presents microarray analysis for several miR-21a $5 p$ target genes. (B) Expression analysis of miR-122-5p and mRNA levels of Fasn and Mttp; and microarray of miR-122-5p target genes. (C) miR-34a-5p gene expression and microarray analysis of targeted genes. (D) Levels of miR-103-3p and Cpt1a by qPCR, and microarray analysis of miR-103-3p targeted genes. CVD conventional diet, HFD high fat and high carbohydrate diet, PFD high fat and high carbohydrate diet + prolonged-release pirfenidone. ${ }^{*} \mathrm{p}<0.05,{ }^{* *} \mathrm{p}<0.01,{ }^{* * *} \mathrm{p}<0.0001$.

Pro-inflammatory cytokines and Collagen 1 were downregulated. As expected, $\operatorname{Il} 1 b(301.1 \pm 44.0$ vs. $1285 \mathrm{a} \pm 86.19, \mathrm{p}<0.0001), \operatorname{ll} 6(250.5 \pm 181.9$ vs $2692 \pm 532.8, \mathrm{p}<0.01)$ and $\operatorname{Tnfa}(279.7 \pm 5.774$ vs. 683.0a \pm 97.78 , $\mathrm{p}<0.05)$ mRNA levels were notably increased in HFD animals compared to CVD group. On the other hand, gene expression of these proinflammatory molecules were definitely diminished in PFD animals (565.3 \pm 85.27 , $1390 \pm 477.3$ and $220.6 \pm 60.19$ with $p<0.0001, p \leq 0.05, p<0.05$; respectively) compared to HFD group. In addition, in Table 2 Collal gene indicates that collagen expression is stimulated in a MAFLD/NASH model, but PFD administration diminish its mRNA levels $(331.1 \pm 64.63$ vs. $97.45 \mathrm{~b} \pm 31.24, \mathrm{p}<0.01)$, This data correlated with previous reports of PFD effects in liver diseases ${ }^{28}$. Fold reduction is indicated in last column of Table 2 when comparing PFD vs HFD groups.

Microarrays analysis. Double-channel chips were analyzed to compare 22,000 genes in mice genomes in the three experimental groups. As shown in Fig. 4A the comparison between CVD animals and HFD mice showed that 82 genes were overexpressed. Pathways associated were: lipid metabolic process (51 genes: Acox2, $A c b d 3, C h 25 h, C p t 1 a$ ), cholesterol metabolic process (9 genes: Ch25h, Vldlr, Apoa4, Mvk), negative regulation of IL6 production (7 genes: Irak3), lipid homeostasis (7 genes: Apoa4, Acadm) and negative regulation of IFNgamma production (7 genes: Il10, Tnfsf4, Il-33). On the other hand, downregulated genes were those involved in: innate immune response (40 genes: Illf6, Traf6), MAPK signaling pathways (23 genes: Smad4, Timp2), metallopeptidase activity (20 genes: Mmp17, Mmp19, Adam17, Adam2), collagen (16 genes: Col3a1, Col1a2, Col11a1), insulin secretion (10 genes: Irs1, Igf1, Irs3) and Ampk signaling pathway (6 genes: Akt1s1) as indicated in Fig. 4B. 
A

CVD
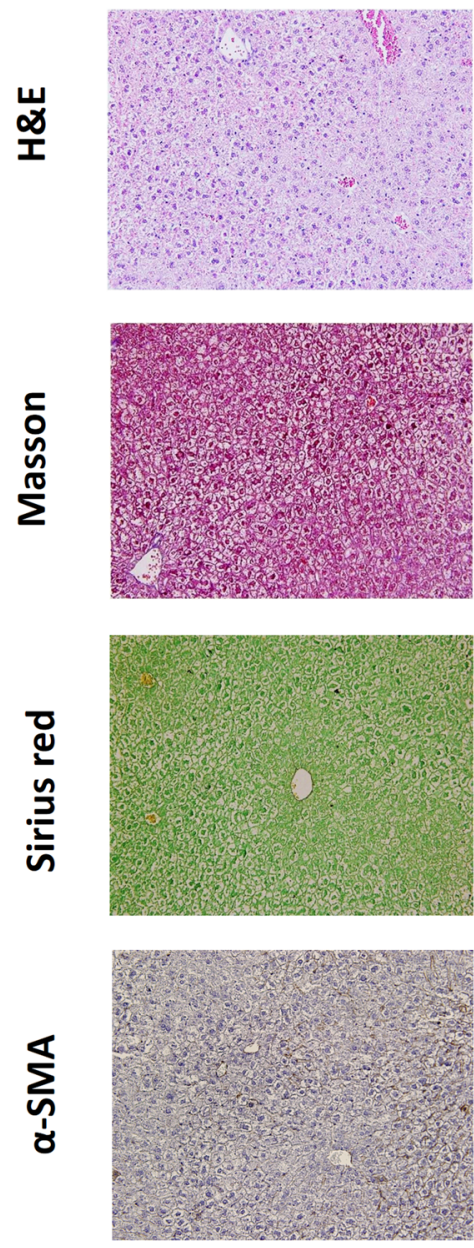

HFD
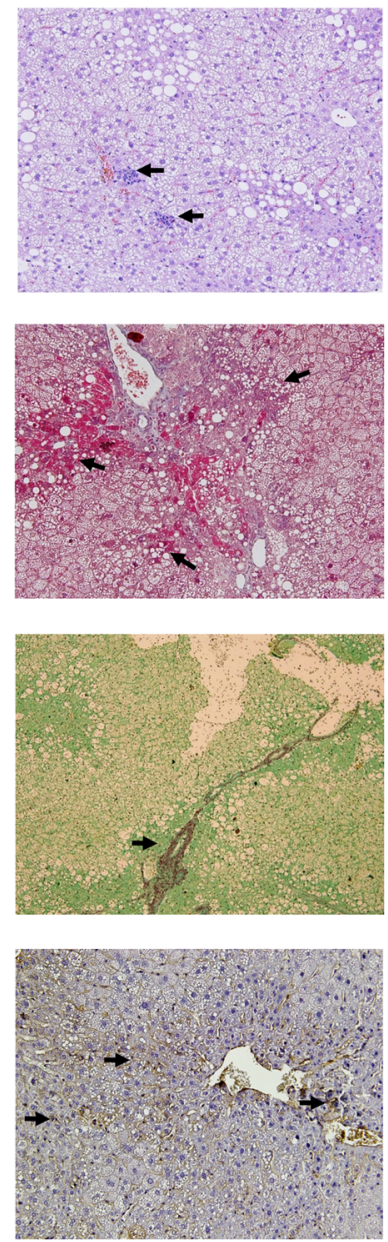

PFD
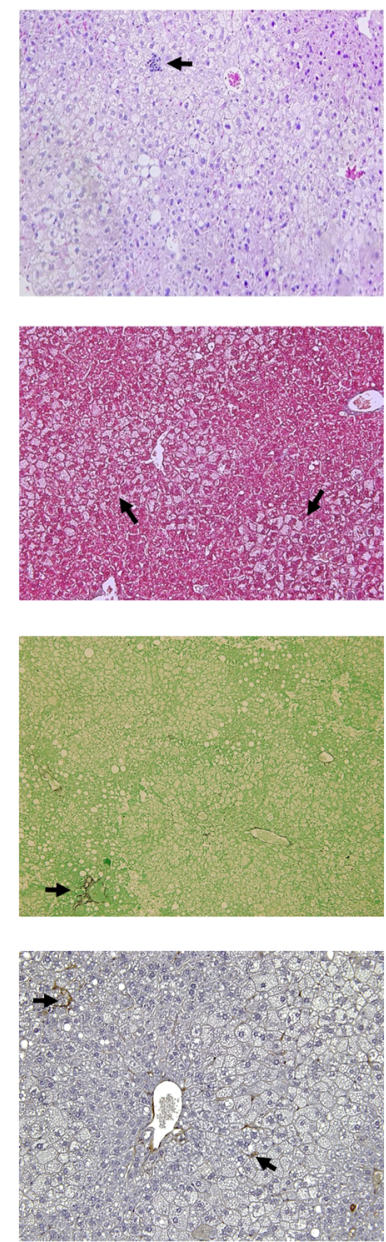

B

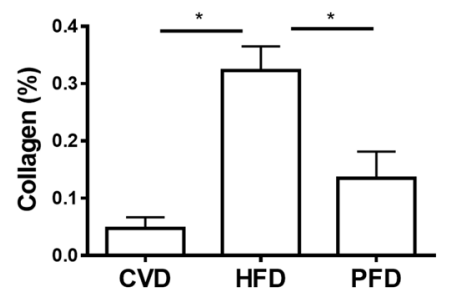

C

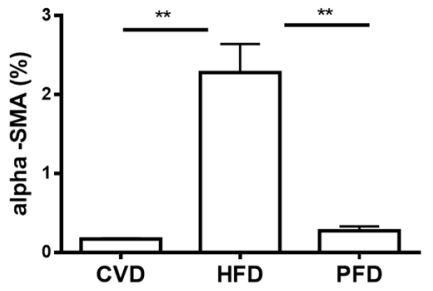

Figure 2. Histological assessment. (A) Microscopic photographs of liver sections. In animals with NASH, an intense inflammation is observed, constituted by neutrophils and mononuclear cells (arrows). Animals treated with PFD showed reduction of inflammatory nodules in H\&E-staining. Masson staining showed initial fibrotic bridges and macrovesicular steatosis in animals fed with HFD. Fibrotic bridges and macrovesicular steatosis were diminished in animals treated with PFD. IHC for alpha-SMA revealed a decreased positivity in treated animals. Sirius red staining indicates a reduction in collagen content in animals receiving PR-PFD. (B) Quantification of collagen staining using Sirius Red in liver tissue $\left({ }^{*} \mathrm{p}<0.05\right)$. Arrows indicate inflammation nodules, lipid vacuoles, fibrosis and collagen bridges. (C)Percentage of alpha-SMA immunoreactivity in treated animals and controls, $\left({ }^{*} \mathrm{p}<0.01\right)$. Photos at $\times 20$ magnification. CVD conventional diet, HFD high fat and high carbohydrate diet, $P F D$ high fat and high carbohydrate diet + prolonged-release pirfenidone. Data are expressed as the media of the group \pm SEM. 
A

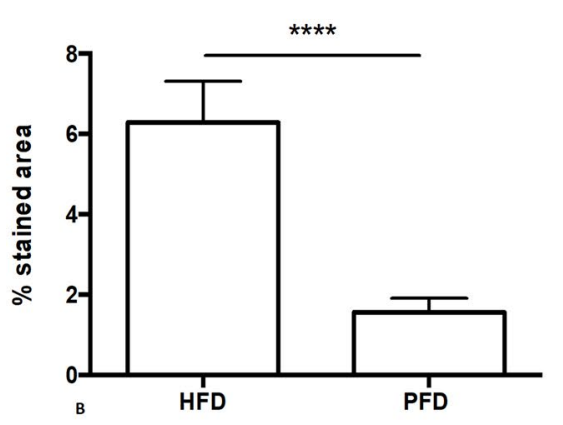

B

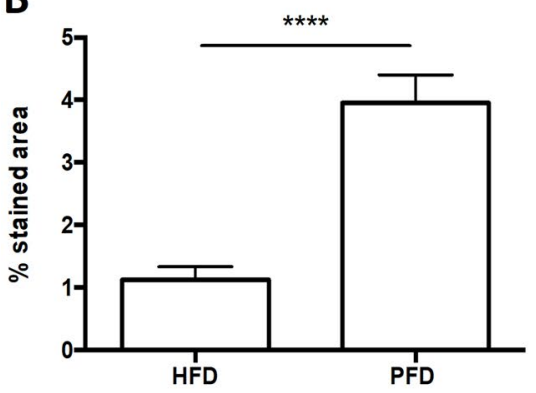

HFD
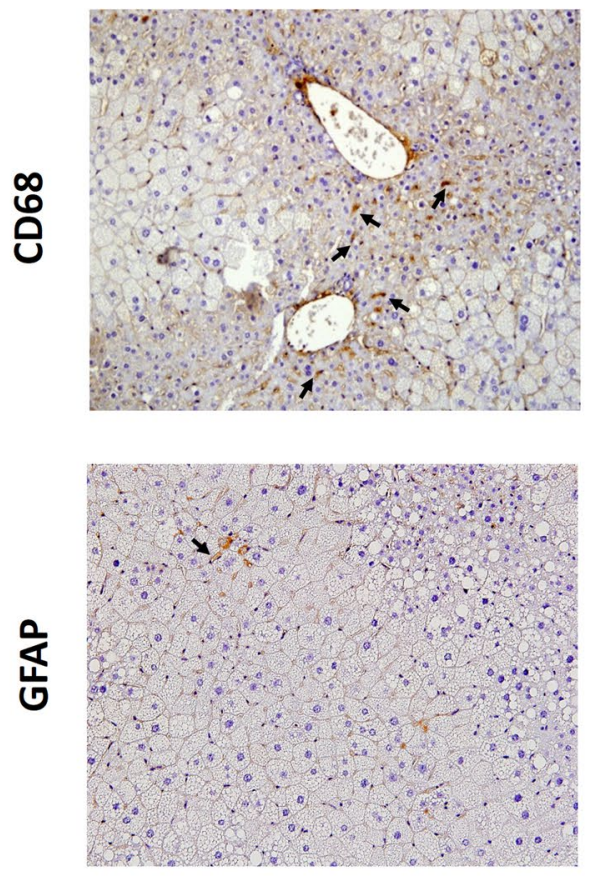

PFD
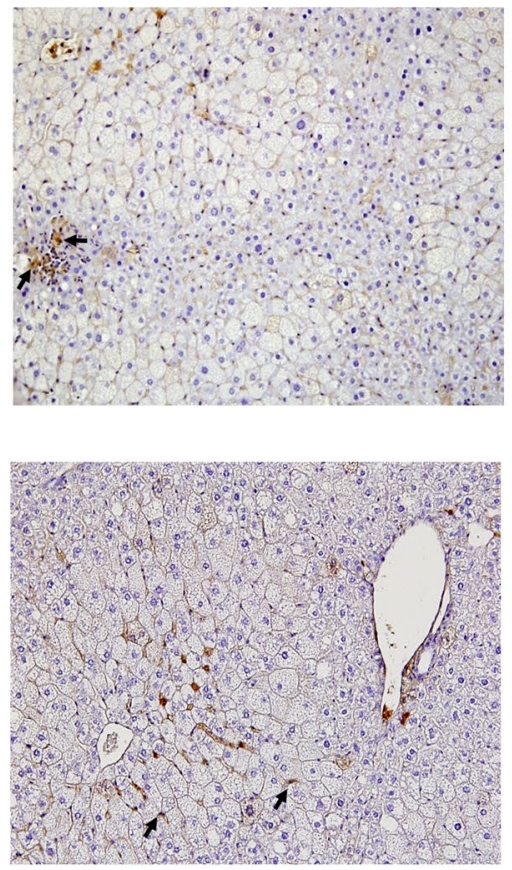

Figure 3. Immunoreactivity against CD68 and GFAP. (A) In animals fed with high fat-high carbohydrate diet an increase in CD68 immunoreactivity was observed. Animals treated with PFD showed a significant reduction in CD68 positive area $\left({ }^{* * *} \mathrm{p}<0.0001\right)$. (B) Quantification of GFAP positive area in liver tissue showed an increased in PFD animals $\left({ }^{* * *} \mathrm{p}<0.0001\right)$. Photographs at $\times 20$ magnification. HFD: high fat and high carbohydrate diet and PFD: high fat and high carbohydrate diet + prolonged-release pirfenidone. Data are expressed as the media of the group \pm SEM.

\begin{tabular}{|l|l|l|l|l|l|}
\hline Gene & CVD & HFD & PFD & Fold change versus HFD & Regulation \\
\hline Il1b & $301.1 \pm 44.0$ & $1285^{\mathrm{a}} \pm 86.19^{* * * *}$ & $565.3^{\mathrm{b}} \pm 85.27^{* * * *}$ & -2.27 & Down \\
\hline Il6 & $250.5 \pm 181.9$ & $2692^{\mathrm{a}} \pm 532.8^{* *}$ & $1390^{\mathrm{b}} \pm 477.3^{*}$ & -1.94 & Down \\
\hline Tnfa & $279.7 \pm 5.774$ & $683.0^{\mathrm{a}} \pm 97.78^{\star}$ & $220.6^{\mathrm{b}} \pm 60.19^{\star}$ & -3.09 & Down \\
\hline Col1a1 & $118.9 \pm 38.03$ & $331.1^{\mathrm{a}} \pm 64.63^{*}$ & $97.45^{\mathrm{b}} \pm 31.24^{* *}$ & -3.40 & Down \\
\hline
\end{tabular}

Table 2. Gene expression of proinflammatory cytokines and Collagen Type I. Data are expressed in mean \pm SEM. CVD conventional diet, HFD high fat and high carbohydrate diet, $P F D$ high fat diet + prolonged release pirfenidone. ${ }^{*} \mathrm{p}<0.05,{ }^{* * *} \mathrm{p}<0.0001$ compared to CVD group and ${ }^{*} \mathrm{p}<0.05,{ }^{* *} \mathrm{p}<0.01,{ }^{* * *} \mathrm{p}<0.0001$ as compared with HFD group. ${ }^{a}$ Significance difference versus CVD group $\left({ }^{*} \mathrm{p}<0.05,{ }^{* *} \mathrm{p}<0.01,{ }^{\star * * *} \mathrm{p}<0.0001\right)$. ${ }^{b}$ Significance difference versus HFD group $\left({ }^{\star} \mathrm{p} \leq 0.05,{ }^{* *} \mathrm{p}<0.01,{ }^{* * *} \mathrm{p}<0.0001\right)$.

When comparing PFD group to HFD group 62 genes were upregulated, and main pathways involved are lipid metabolism (33 genes: Acaca, Apoa1, Acox1). Response to oxidative stress (16 genes: Mmp14, Prdx1), lipid transport (15 genes: Lbp, Hdl, Apoc4), insuling signaling pathway (14 genes: Cftr/Mrp, Abcc8), Baiap2l1, Atf2). Ppar signaling pathway (5 genes: Crebbp), antioxidant activity (5 genes: Gpx3, Gsr, Fabp1), cholesterol transport activity (4 genes: Scp2, Apoa1, Apoa5) and fatty acid desaturase, type 1, C-terminal (3 genes: $S c d 1$, Fads1, $S c d 2, S c d 3$ ) (Fig. 4C). Genes which were downregulated are implicated in innate immune response (34 genes: Ifna2, Defb41, Il2), inflammatory response (20 genes: Il12b, Ifng, Il2). Also, downregulation was seen in collagen metabolism pathways (19 genes: Col13a1, Col14a1), insulin resistance (14 genes: Irs1, Tnfrsf1a), lipid biosynthesis (13 genes: Ch25h, Srebf2, Hacd4), negative regulation of Nfkb transcription factor activity (10 genes: Cmklr1, Nfkbia, Pias4), Finally, oxidative stress-induced gene expression via NRF2 (5 genes: Hmox2, Prkca) and hepatic stellate cell activation (4 genes: Dgat1) (Fig. 4D) were suppressed as well.

\section{Discussion}

Several studies have shown that specific miRNAs play a key role in the progression of metabolic associated fatty liver disease (MAFLD), mostly using high-fat diet animal models. Therefore, modulation of miRNA expression could be a potential therapeutic target for the treatment of this disease because miRNAs regulate lipid synthesis, glucose and fatty acid catabolism, inflammation, proliferation, apoptosis and necrosis; all processes epigenetically 
A

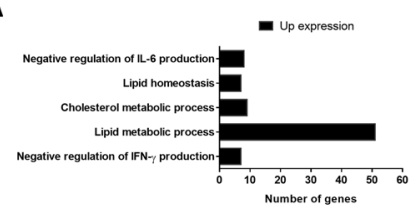

C

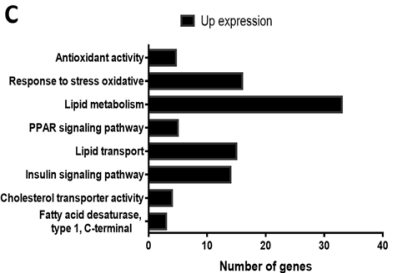

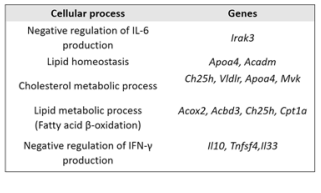

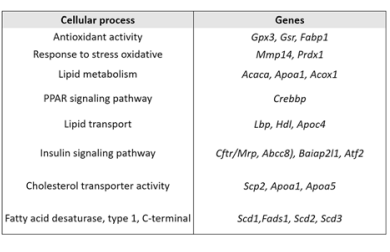

B

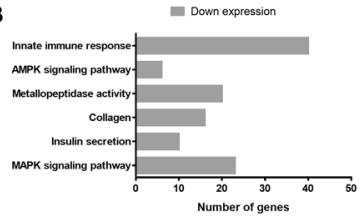

D

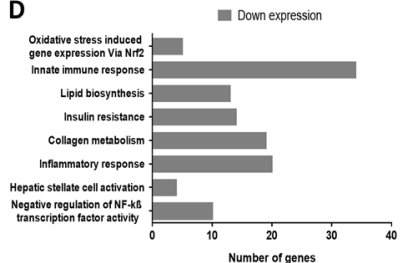

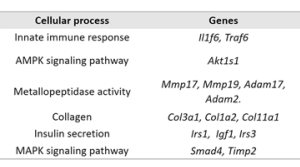

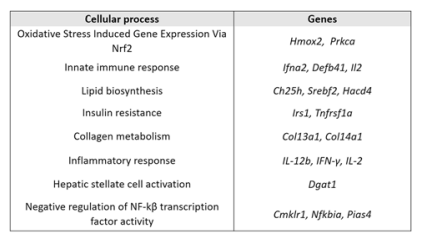

Figure 4. Microarray analysis of cellular pathways with gene dysregulation. CVD compared to HFD: (A) pathways with upregulated genes and (B) cellular tracks with decreased gene expression. PFD compared to HFD: (C) shows cell processes with gene overexpression and (D) metabolic pathways with decreased gene expression. CVD conventional diet, HFD high fat and high carbohydrate diet, PFD high fat and high carbohydrate diet + prolonged-release pirfenidone.

deregulated in $\mathrm{NASH}^{15,23}$. A comprehensive literature search was carried out to select some of the most representative miRNAs for each of the key processes found to be dysregulated in non-alcoholic steatohepatitis, which are inflammation, fibrosis, steatosis and insulin resistance. Therefore, alterations on miRNAs target genes involved in liver energy metabolism, inflammation, cell regeneration and fibrogenic signaling; driving the progression from MAFLD to NASH were considered. Our study is the first to show that miR-21a-5p, miR-122-5p, miR-34a-5p and miR-103-3p expression and numerous of their target genes like, Srebfl, Tgfbl, Fasn and Cpt1a are modified by pirfenidone treatment in a MAFLD/NASH model as part of an improvement in molecular, histopathological and biochemical parameters. miR-21a-5p has been extensively studied in liver diseases, as well as its main target genes. miR-21a-5p hepatic expression is increased in animal models and in patients with MAFLD/NASH ${ }^{38-40}$. Similarly, we found a significant increased expression of miR-21a-5p induced by high-fat diet in C57BL/6J mice. miR-21a-5p is consider a profibrogenic miRNA by its effect on Smad7 and also participates in the accumulation of liver lipids by interacting with various factors, such as sterol regulatory element binding transcription factor 1 (Srebf1), 3-hydroxy-3-methylglutaryl-coenzyme A reductase (Hmgcr) and protein binding to fatty acids 7 $(\text { Fabp7 })^{41-43}$. We found that miR-21a-5p expression was decreased in PFD group, as well as, Srebf1 and Tgfb1. miR-21a-5p hepatic diminution has been associated with improved glucose tolerance and insulin sensitivity, in addition to prevent hepatic steatosis and fatty acid absorption ${ }^{44}$. $\mathrm{Tg} f b$ signaling is crucial for fibrogenesis as has been convincingly demonstrated in numerous studies ${ }^{45,46}$. In our study, $\mathrm{Tg} f b 1$ decrease in PFD group associates with decreased expression of Colla1. These results correlated with histological analysis displaying a reduction in the number of fibrotic bridges, periportal and pericentral fibrosis and percentage of collagen stained area in PFD group. We also found a faded alpha-SMA positive area, indicating a contracted activation of HSCs due to PFD treatment; which correlates with an increase in GFAP (Glial fibrillary acidic protein) quiescent marker in HSCs in these group. As stated by Zisser et al. and others, quiescent HSCs are characterized by the cytoplasmic storage of vitamin A and markers include, PPARg, GFAP, and BAMBI ${ }^{47-49}$. These observed effects are caused by PFD; a drug widely known for reducing fibrosis and collagen deposition in various organs and diseases, including MAFLD models ${ }^{28,50,51}$. Nuclear receptor peroxisome proliferation-activated receptor alpha (Ppara) is target gene of miR-21a-5p. A study has shown that miR-21a-5p is increased in NASH patients, resulting in diminished expression of $P_{p a r a}{ }^{52}$. In this way, miR-21a-5p contributes to cell injury, inflammation, and fibrosis, through its inhibition of the PPARA signaling pathway ${ }^{23}$. Also, Sandoval-Rodriguez et al. had previously reported that protein expression of Ppara is diminished in a mouse MAFLD/NASH model and restored to similar levels than CVD in animals treated with PFD ${ }^{51}$.

miR-122-5p is one of the most abundant miRNAs in the liver, it constitutes $70 \%$ of all hepatic miRNAs. It has been reported that miR-122-5p is overexpressed in liver tissue of C57BL/6 mice with MAFLD, and in HepG2 and Huh-7 cells exposed to FFA. These in vitro models present an excess of lipid accumulation and triglycerides secretion, decreased expression of Sirt 1 and genes related to lipogenesis ${ }^{53}$. Similarly, in the present study, we found that miR-122-5p expression was remarkably elevated in HFD mice as determined by qRT-PCR. Conversely, former studies have shown that miR-122-5p is decreased in MAFLD models induced by HFD. Also, reduced levels of miR-122-5p were identified in liver biopsies of patients with NASH, at the most severe stage of the MAFLD spectrum, compared to control group ${ }^{39}$. A possible explanation for these divergent results is the diverse animals' strains and variety of conditions of MAFLD induction, as well as the different co-morbidities and severity in patients with MAFLD.

miR-122-5p has been reported to be expressed primarily in hepatocytes and to target multiple enzymes in lipid metabolism, including fatty acid synthase (Fasn), 3-hydroxy-3-methyl-glutarylcoenzyme A reductase (Hmgcr), and Srebf1 and Srebf $2^{54}$. We found that miR-122-5p reduced expression in PFD group correlates with Fasn mRNA reduced expression in microarray. Fasn catalyzes the last step in fatty acid biosynthesis and therefore it is an important determinant of maximal liver ability to generate fatty acids by novo lipogenesis ${ }^{14}$. 
Recently, miR-34a-5p has been shown to be specifically modulated in liver diseases. Circulating miR-34a-5p levels are high in patients with MAFLD and in animal models of steatosis ${ }^{55}$. Similarly, our data showed a higher hepatic miR-34a-5p expression, while PFD administration attenuates its induction. Also, a decrease in the expression of miR-34a-5p and an increase in its target genes Sirt1, Ppara, and Insig2 has been reported when green tea was used to protect against MAFLD development in a rodent model ${ }^{56}$. Hnf $4 a$ is a target gene of miR-34a-5p known to modulate the regulatory elements of promoters and enhancers of genes involved in the metabolism of cholesterol, fatty acids and glucose. In microarray analysis, our data showed increased Hnf $4 a$ expression by PFD treatment. Specifically in the liver, $\mathrm{Hnf} 4 \mathrm{a}$ activates hepatic gluconeogenesis and regulates expression of several genes, including apolipoproteins ${ }^{57}$.

miR-103-3p has been shown to regulate insulin sensitivity and glucose homeostasis, previous studies have found that miR-103-3p expression was increased in the liver of patients with MAFLD; as well as, in vitro and in vivo experimental models of MAFLD/NASH ${ }^{58-60}$. In our study, miR-103-3p expression was also detected increased due to high-fat diet induction of NASH. Silencing miR-103-3p improved insulin resistance. In contrast, the gain of function of miR-103-3p in the liver was sufficient to induce insulin resistance ${ }^{21}$. Other studies confirmed that high expression of miR-103-3p led to insulin resistance by decreasing the expression of caveolin-1, which is a direct target of miR-103-3p and a critical regulator of insulin receptor ${ }^{21,22}$. These data correlate with the effects seen after PFD treatment, since ITT was improved and miR-103 expression was reduced. In accordance with the miRWalk database 2.0, Srebf1 is a predicted miR-103-3p target gene. Srebf1 is one of the main regulators of de novo lipogenesis in MAFLD, and Srebf1 overexpression contributed predominantly to lipids accumulation ${ }^{61}$. In resveratrol-treated obese rats, a significant decreased expression of miR-103-3p was reported, as well as, a decline in Srebf1 protein expression ${ }^{60}$. According to these facts, in PFD animals we observed the same pattern regarding these molecules. In agreement to the miRWalk database 2.0, carnitine palmitoyltransferase la (Cpt1a) is a predicted target gene for miRNA-103-3p. Cpt1a is a key enzyme involved in mitochondrial $\beta$-oxidation, catalyzes the transfer of acyl groups from fatty acyl-CoAs to carnitine in the mitochondrial membrane for the translocation into the intermembrane space ${ }^{62,63}$. Cpt1a expression is decreased in our MAFLD model induced by high-fat diet, while increased in PFD group.

Key cellular processes are found to be altered in the development of non-alcoholic steatohepatitis (NASH). Using microarray analyses, we examined genes involved in lipid metabolism, inflammation, oxidative stress, fibrosis, insulin resistance, and how these pathways or cellular metabolism were modified in NASH and after administration of prolonged-release pirfenidone.

When comparing PFD group to HFD group, we found a decreased expression in genes involved in hepatic stellate cells activation, collagen metabolic processes and transforming growth factor $\beta$ receptor binding, events involved in fibrosis progression, which is coherent with the well-known antifibrotic effect of pirfenidone in diverse fibrotic diseases ${ }^{27,28,64,65}$.

Particularly in NASH related to MAFLD, recently Komiya et al. and Sandoval-Rodriguez et al. found that pirfenidone improves liver fibrosis in mice models ${ }^{50,51}$. Gutierrez-Cuevas et al. also found in a model of MAFLD induced by High fat diet that PFD showed beneficial effects in cardiac fibrosis ${ }^{66}$.

Insulin resistance is a characteristic feature of MAFLD that contributes to its pathogenesis. Under conditions of insulin resistance, abnormally high levels of insulin are required to metabolize glucose and inhibit hepatic glucose production effectively due to reduced insulin sensitivity in peripheral tissues. Insulin resistance stimulates the pancreas to increase insulin secretion from the portal vein, leading to higher levels of insulin in the liver than in the periphery. High concentrations of hepatic glucose and plasmatic insulin are recognized as biomarkers of hepatic insulin resistance. Elevated fasting glucose levels are due to hepatic insulin resistance, while elevated concentrations of free fatty acids occur ${ }^{67}$. Microarrays showed a decrease expression in genes that participate in insulin resistance and in insulin secretion in the PFD group compared to HF group, which indicates that hyperinsulinemia might be caused by a high fat diet.

Activation of innate response of the immune system during NASH occurs mainly due to excessive accumulation of lipids that leads to hepatocytes damage, activating an inflammatory response that worsens the progress of liver disease. Inflammation is characterized by $T n f a, I l 1 b$, as well as reactive oxygen species production ${ }^{68}$. Our data indicates a decrease expression in genes involved in the innate response of the immune system in PFD mice versus HFD group. Extensive experimental and clinical data support a central role for macrophages in the development and progression of NASH. Liver-resident Kupffer cells initiate inflammation and help recruit blood-derived monocytes ${ }^{69}$. Macrophage polarization to M1 phenotype (proinflammatory) contribute to NASH $\operatorname{progression}^{70}$. Here, we found a significant reduction in immunoreactivity of CD68 marker in PFD treated animals. These data suggest that pirfenidone is able to reduce inflammatory response activation, correlating with the already reported anti-inflammatory properties of $\mathrm{PFD}^{28,71}$.

$N f k b$ is a transcription factor involved in innate and adaptive immune responses, as well as in a number of pathological processes, such as inflammation. Under normal conditions, $N f k b$ is sequestered in the cytoplasm and binds to IKB proteins, inhibiting the nuclear localization of NFKB. NFKB activation is normally moderate, whereas, under conditions of insulin resistance, its expression in the liver is greatly increased. $N f k b$ translocation to the nucleus leads to upregulation of target genes encoding inflammatory mediators, such as Tnfa and $I l 6^{72}$. We found a decrease expression of genes related to the inflammatory response in PFD mice compared to the HFP group, which could be linked to a decrease in the negative regulation of $N f k b$ activity.

We also found a decreased expression in genes that participate in the metabolic process of lipids and fatty acids, mainly of genes that participate in lipogenesis in PFD animals compared to HFD group. On the other hand, microarrays showed an increase in the expression of genes related with activity of the cholesterol transporter, the transport of lipids and type 1 fatty acid desaturase. Previous studies have reported changes in the expression of liver desaturases during the development of MAFLD/NASH, it was found that a significant decrease in the activity of Fads1 in the progression to $\mathrm{NASH}^{63}$. Regarding cholesterol transport genes, hepatic accumulation of 
free cholesterol has been shown to be an outstanding feature in MAFLD, which correlates with the histological severity of the disease. Additionally, epidemiological studies have identified dietary cholesterol intake as a factor related to the risk and severity of MAFLD. Transport of lipids is important, since an excessive flow of free fatty acids into the liver can cause NASH. Diet is an important component in the development of MAFLD, since 15\% of triglycerides in the liver have the diet as their source ${ }^{73}$. Fatty acids in the diet are involved in liver lipogenesis and could play a dual role in the pathogenesis of liver steatosis, since they are involved in its development and in its prevention or reversal depending of the amount of omega-fatty acids.

Lipotoxicity, arising from hepatic fat excess, leads to mitochondrial dysfunction associated with an elevated ability to oxidize fatty acids, resulting in the production of reactive oxygen species (ROS). In transcriptome analysis, a decreased expression in genes involved in the response to oxidative stress was present in PFD group compared to HFD group. MAFLD causes overexpression of antioxidant agents, but the unbalanced production of ROS promotes oxidative stress. Pirfenidone has been reported to have antioxidant capacity ${ }^{74,75}$ and this effect correlates with expected benefits in this MALFD model. Oxidative stress can induce hepatocellular damage by inhibiting enzymes involved in mitochondrial respiratory chain and inactivation of glyceraldehyde3 -phosphate dehydrogenase ${ }^{76}$. Furthermore, ROS cause lipid peroxidation and cytokine production, which contributes to hepatocellular injury and fibrosis, promoting the progression from simple steatosis to nonalcoholic steatohepatitis ${ }^{68}$. Oxidative stress in MAFLD induces hepatic stellate cell activation, the most important producer of extracellular matrix ${ }^{77,78}$. In summary, increased expression of miR-21a-5p, miR-34a-5p, miR-122-5p and miR-103-3p in this MAFLD/NASH model was reversed with prolonged-release pirfenidone. MAFLD/NASH group compared to conventional diet control revealed modifications in gene metabolic pathways implicated in lipid metabolic process, inflammatory response, antioxidant activity and insulin resistance; PR-PFD seems to reverse these modifications.

Received: 27 February 2021; Accepted: 24 May 2021

Published online: 03 June 2021

\section{References}

1. Younossi, Z. M. et al. Global epidemiology of nonalcoholic fatty liver disease-meta-analytic assessment of prevalence, incidence, and outcomes. Hepatology 64, 73-84 (2016).

2. Tilg, H. \& Effenberger, M. From NAFLD to MAFLD: When pathophysiology succeeds. Nat. Rev. Gastroenterol. Hepatol. 17, 387-388 (2020).

3. Eslam, M., Sanyal, A. J. \& George, J. MAFLD: A consensus-driven proposed nomenclature for metabolic associated fatty liver disease. Gastroenterology 158, 1999-2014.e1991 (2020).

4. Fabbrini, E., Sullivan, S. \& Klein, S. Obesity and nonalcoholic fatty liver disease: Biochemical, metabolic, and clinical implications. Hepatology 51, 679-689 (2010).

5. Perumpail, B. J. et al. Clinical epidemiology and disease burden of nonalcoholic fatty liver disease. World J. Gastroenterol. 23, 8263-8276 (2017).

6. Younossi, Z. et al. Global burden of NAFLD and NASH: Trends, predictions, risk factors and prevention. Nat. Rev. Gastroenterol. Hepatol. 15, 11-20 (2018).

7. Estes, C. \& Razavi, H. Modeling the epidemic of nonalcoholic fatty liver disease demonstrates an exponential increase in burden of disease. Hepatology 67, 123-133 (2018).

8. Lizardi-Cervera, J., Laparra, D. I., Chavez-Tapia, N. C., Ostos, M. E. \& Esquivel, M. U. Prevalence of NAFLD and metabolic syndrome in asymtomatics subjects. Rev. Gastroenterol. Mex. 71, 453-459 (2006).

9. Saab, S., Manne, V., Nieto, J., Schwimmer, J. B. \& Chalasani, N. P. Nonalcoholic fatty liver disease in latinos. Clin. Gastroenterol. Hepatol. 14, 5-12 (2016) (quiz e19-10).

10. Brunt, E. M. et al. Nonalcoholic fatty liver disease. Nat. Rev. Dis. Primers 1, 15080 (2015).

11. Szabo, G. \& Bala, S. MicroRNAs in liver disease. Nat. Rev. Gastroenterol. Hepatol. 10, 542-552 (2013).

12. Bartel, D. P. MicroRNAs: Genomics, biogenesis, mechanism, and function. Cell 116, 281-297 (2004).

13. He, Z., Hu, C. \& Jia, W. miRNAs in non-alcoholic fatty liver disease. Front Med. 10, 389-396 (2016).

14. Szabo, G. \& Csak, T. Role of microRNAs in NAFLD/NASH. Dig. Dis. Sci. 61, 1314-1324 (2016).

15. Baffy, G. MicroRNAs in nonalcoholic fatty liver disease. J. Clin. Med. 4, 1977-1988 (2015).

16. Pirola, C. J. et al. Circulating microRNA signature in non-alcoholic fatty liver disease: From serum non-coding RNAs to liver histology and disease pathogenesis. Gut 64, 800-812 (2015).

17. Moore, K. J., Rayner, K. J., Suárez, Y. \& Fernández-Hernando, C. The role of microRNAs in cholesterol efflux and hepatic lipid metabolism. Annu. Rev. Nutr. 31, 49-63 (2011).

18. Bandiera, S., Pfeffer, S., Baumert, T. F. \& Zeisel, M. B. miR-122-a key factor and therapeutic target in liver disease. J. Hepatol. 62, 448-457 (2015).

19. Ding, J. et al. Effect of miR-34a in regulating steatosis by targeting PPARalpha expression in nonalcoholic fatty liver disease. Sci. Rep. 5, 13729 (2015).

20. Xu, Y. et al. A metabolic stress-inducible miR-34a-HNF4alpha pathway regulates lipid and lipoprotein metabolism. Nat. Commun. 6, $7466(2015)$.

21. Xu, Q., Li, Y., Shang, Y.-F., Wang, H.-L. \& Yao, M.-X. miRNA-103: Molecular link between insulin resistance and nonalcoholic fatty liver disease. World J. Gastroenterol. 21, 511-516 (2015).

22. Trajkovski, M. et al. MicroRNAs 103 and 107 regulate insulin sensitivity. Nature 474, 649-653 (2011).

23. Loyer, X. et al. Liver microRNA- 21 is overexpressed in non-alcoholic steatohepatitis and contributes to the disease in experimental models by inhibiting PPARalpha expression. Gut 65, 1882-1894 (2016).

24. Krichevsky, A. M. \& Gabriely, G. miR-21: A small multi-faceted RNA. J. Cell Mol. Med. 13, 39-53 (2009)

25. Caligiuri, A., Gentilini, A. \& Marra, F. Molecular pathogenesis of NASH. Int. J. Mol. Sci. 17, 20 (2016).

26. Ocker, M. Challenges and opportunities in drug development for nonalcoholic steatohepatitis. Eur. J. Pharmacol. 870, 172913 (2020).

27. Di Sario, A. et al. The anti-fibrotic effect of pirfenidone in rat liver fibrosis is mediated by downregulation of procollagen alpha1(I), TIMP-1 and MMP-2. Dig. Liver Dis. 36, 744-751 (2004).

28. Lopez-de la Mora, D. A. et al. Role and new insights of pirfenidone in fibrotic diseases. Int. J. Med. Sci. 12, 840-847 (2015). 
29. Poo, J. L. B. R. et al. Pharmacokinetics of the antifibrotic drug pirfenidone in Child-Pugh A and B cirrhotic patients compared to healthy age-matched controls. J. Hepatol. 64, S213-S424 (2016).

30. Allison, D. B., Paultre, F., Maggio, C., Mezzitis, N. \& Pi-Sunyer, F. X. The use of areas under curves in diabetes research. Diabetes Care 18, 245-250 (1995).

31. Matthews, D. R. et al. Homeostasis model assessment: Insulin resistance and beta-cell function from fasting plasma glucose and insulin concentrations in man. Diabetologia 28, 412-419 (1985).

32. Chomczynski, P. \& Sacchi, N. Single-step method of RNA isolation by acid guanidinium thiocyanate-phenol-chloroform extraction. Anal. Biochem. 162, 156-159 (1987).

33. Schmittgen, T. D. \& Livak, K. J. Analyzing real-time PCR data by the comparative CT method. Nat. Protoc. 3, 1101-1108 (2008).

34. Brown, R. A. M. et al. Total RNA extraction from tissues for microRNA and target gene expression analysis: Not all kits are created equal. BMC Biotechnol. 18, 16 (2018).

35. Schmittgen, T. D. \& Livak, K. J. Analyzing real-time PCR data by the comparative C(T) method. Nat. Protoc. 3, 1101-1108 (2008).

36. Unidad de Microarreglos de DNA-Instituto de Fisiología Celular, U. Microarreglos de ratón, http://microarrays.ifc.unam.mx/ (Updated).

37. da Huang, W., Sherman, B. T. \& Lempicki, R. A. Systematic and integrative analysis of large gene lists using DAVID bioinformatics resources. Nat. Protoc. 4, 44-57 (2009).

38. Loyer, X. et al. Liver microRNA-21 is overexpressed in non-alcoholic steatohepatitis and contributes to the disease in experimental models by inhibiting PPARa expression. Gut 65, 1882-1894 (2016).

39. Cheung, O. et al. Nonalcoholic steatohepatitis is associated with altered hepatic MicroRNA expression. Hepatology 48, 1810-1820 (2008).

40. Liu, J. et al. A circulating microRNA signature as noninvasive diagnostic and prognostic biomarkers for nonalcoholic steatohepatitis. BMC Genom. 19, 188 (2018).

41. Wu, H., Ng, R., Chen, X., Steer, C. J. \& Song, G. MicroRNA-21 is a potential link between non-alcoholic fatty liver disease and hepatocellular carcinoma via modulation of the HBP1-p53-Srebp1c pathway. Gut 65, 1850-1860 (2016).

42. Sun, C. et al. miR-21 regulates triglyceride and cholesterol metabolism in non-alcoholic fatty liver disease by targeting HMGCR. Int. J. Mol. Med. 35, 847-853 (2015).

43. Ahn, J., Lee, H., Jung, C. H. \& Ha, T. Lycopene inhibits hepatic steatosis via microRNA-21-induced downregulation of fatty acidbinding protein 7 in mice fed a high-fat diet. Mol. Nutr. Food Res. 56, 1665-1674 (2012).

44. Calo, N. et al. Stress-activated miR-21/miR-21* in hepatocytes promotes lipid and glucose metabolic disorders associated with high-fat diet consumption. Gut 65, 1871-1881 (2016).

45. Yang, L. et al. Transforming growth factor beta signaling in hepatocytes participates in steatohepatitis through regulation of cell death and lipid metabolism in mice. Hepatology 59, 483-495 (2014).

46. Zhang, T., Yang, Z., Kusumanchi, P., Han, S. \& Liangpunsakul, S. Critical role of microRNA-21 in the pathogenesis of liver diseases. Front Med. 7, 20 (2020).

47. Shang, L., Hosseini, M., Liu, X., Kisseleva, T. \& Brenner, D. A. Human hepatic stellate cell isolation and characterization. J. Gastroenterol. 53, 6-17 (2018).

48. Friedman, S. L. Hepatic stellate cells: Protean, multifunctional, and enigmatic cells of the liver. Physiol. Rev. 88, 125-172 (2008).

49. Zisser, A., Ipsen, D. H. \& Tveden-Nyborg, P. Hepatic stellate cell activation and inactivation in NASH-fibrosis-roles as putative treatment targets?. Biomedicines $\mathbf{9}, 365$ (2021).

50. Komiya, C. et al. Antifibrotic effect of pirfenidone in a mouse model of human nonalcoholic steatohepatitis. Sci. Rep. 7, 44754 (2017).

51. Sandoval-Rodriguez, A. et al. pirfenidone is an agonistic ligand for PPARa and improves NASH by activation of SIRT1/LKB1/ pAMPK. Hepatol. Commun. 4, 434-449 (2020).

52. Rodrigues, P. M., Rodrigues, C. M. P. \& Castro, R. E. Modulation of liver steatosis by miR-21/PPARa. Cell Death Discov. 4, 9 (2018),

53. Long, J. K., Dai, W., Zheng, Y. W. \& Zhao, S. P. miR-122 promotes hepatic lipogenesis via inhibiting the LKB1/AMPK pathway by targeting Sirt1 in non-alcoholic fatty liver disease. Mol. Med. 25, 26 (2019).

54. Dorn, C. et al. Expression of fatty acid synthase in nonalcoholic fatty liver disease. Int. J. Clin. Exp. Pathol. 3, 505-514 (2010).

55. Xu, Y. et al. A metabolic stress-inducible miR-34a-HNF4a pathway regulates lipid and lipoprotein metabolism. Nat. Commun. 6 , 7466 (2015)

56. Torres, L. F., Cogliati, B. \& Otton, R. Green tea prevents NAFLD by modulation of miR-34a and miR-194 expression in a high-fat diet mouse model. Oxid. Med. Cell. Longev. 2019, 4168380 (2019).

57. Baciu, C. et al. Systematic integrative analysis of gene expression identifies HNF4A as the central gene in pathogenesis of nonalcoholic steatohepatitis. PLoS One 12, e0189223 (2017).

58. Soronen, J. et al. Novel hepatic microRNAs upregulated in human nonalcoholic fatty liver disease. Physiol. Rep. 4, e12661 (2016).

59. Jin, X. et al. Transition from hepatic steatosis to steatohepatitis: Unique microRNA patterns and potential downstream functions and pathways. J. Gastroenterol. Hepatol. 27, 331-340 (2012).

60. Gracia, A. et al. Are miRNA-103, miRNA-107 and miRNA-122 involved in the prevention of liver steatosis induced by resveratrol?. Nutrients 9, 360 (2017).

61. Higuchi, N. et al. Liver X receptor in cooperation with SREBP-1c is a major lipid synthesis regulator in nonalcoholic fatty liver disease. Hepatol. Res. 38, 1122-1129 (2008).

62. Koo, S.-H. Nonalcoholic fatty liver disease: Molecular mechanisms for the hepatic steatosis. Clin. Mol. Hepatol. 19, 210-215 (2013).

63. Ipsen, D. H., Lykkesfeldt, J. \& Tveden-Nyborg, P. Molecular mechanisms of hepatic lipid accumulation in non-alcoholic fatty liver disease. Cell Mol. Life Sci. 75, 3313-3327 (2018).

64. Garcia, L. et al. Pirfenidone effectively reverses experimental liver fibrosis. J. Hepatol. 37, 797-805 (2002).

65. Zhao, X. Y., Zeng, X., Li, X. M., Wang, T. L. \& Wang, B. E. Pirfenidone inhibits carbon tetrachloride- and albumin complex-induced liver fibrosis in rodents by preventing activation of hepatic stellate cells. Clin. Exp. Pharmacol. Physiol. 36, 963-968 (2009).

66. Gutiérrez-Cuevas, J. et al. Prolonged-release pirfenidone prevents obesity-induced cardiac steatosis and fibrosis in a mouse NASH model. Cardiovasc. Drugs Ther. 20, 20 (2020).

67. Chen, Z., Yu, R., Xiong, Y., Du, F. \& Zhu, S. A vicious circle between insulin resistance and inflammation in nonalcoholic fatty liver disease. Lipids Health Dis. 16, 203-203 (2017).

68. Masarone, M., Rosato, V., Dallio, M. \& Gravina, A. G. Role of oxidative stress in pathophysiology of nonalcoholic fatty liver disease. Oxid. Med. Cell Longev. 2018, 9547613 (2018).

69. Kazankov, K. \& Jørgensen, S. M. D. The role of macrophages in nonalcoholic fatty liver disease and nonalcoholic steatohepatitis. Nat. Rev. 16, 145-159 (2019).

70. Grunhut, J. et al. Macrophages in nonalcoholic steatohepatitis: Friend or foe?. Eur. Med. J. Hepatol. 6, 100-109 (2018).

71. Chen, G., Ni, Y. \& Nagata, N. Pirfenidone prevents and reverses hepatic insulin resistance and steatohepatitis by polarizing M2 macrophages. Lab. Invest. 99, 1335-1348 (2019).

72. Kosmalski, M., Mokros, $€$, Kuna, P., Witusik, A. \& Pietras, T. Changes in the immune system-the key to diagnostics and therapy of patients with non-alcoholic fatty liver disease. Cent. Eur. J. Immunol. 43, 231-239 (2018).

73. Nakamuta, M. et al. Evaluation of fatty acid metabolism-related gene expression in nonalcoholic fatty liver disease. Int. J. Mol. Med. 16, 631-635 (2005). 
74. Salazar-Montes, A., Ruiz-Corro, L., Lopez-Reyes, A., Castrejon-Gomez, E. \& Armendariz-Borunda, J. Potent antioxidant role of pirfenidone in experimental cirrhosis. Eur. J. Pharmacol. 595, 69-77 (2008).

75. Liu, Y., Lu, F., Kang, L., Wang, Z. \& Wang, Y. Pirfenidone attenuates bleomycin-induced pulmonary fibrosis in mice by regulating Nrf2/Bach1 equilibrium. BMC Pulm. Med. 17, 63 (2017).

76. Rolo, A. P., Teodoro, J. S. \& Palmeira, C. M. Role of oxidative stress in the pathogenesis of nonalcoholic steatohepatitis. Free Radic. Biol. Med. 52, 59-69 (2012).

77. Palladini, G. et al. Fatty acid desaturase involvement in non-alcoholic fatty liver disease rat models: Oxidative stress versus metalloproteinases. Nutrients 11, 799 (2019).

78. Arroyave-Ospina, J. C., Wu, Z. \& Geng, Y. Role of oxidative stress in the pathogenesis of non-alcoholic fatty liver disease: Implications for prevention and therapy. Antioxidants 10, 174 (2021).

\section{Acknowledgements}

Rebeca Escutia-Gutiérrez would like to thank CONACYT for the scholarship granted to obtain her PhD.

\section{Author contributions}

R.E.-G.: sample processing, therapy administration, animal model, miRNA expression analysis, data analysis and article writing. J.S.R.-S.: animal models and laboratory studies. C.A.M.-M.: gene expression analysis and data analysis. J.G.-B.: sample processing, therapy administration and statistics. A.S.-G.: critical intellectual input. A.S.R.: article writing, data analysis, methodological analysis and supervision. J.A.-B.: article writing, data analysis and supervision. All authors have reviewed and approved the final version of the manuscript.

\section{Competing interests}

Dr. Juan Armendariz-Borunda is a consultant for Cell Pharma S.A. de C.V. The other authors declare no conflict of interest.

\section{Additional information}

Supplementary Information The online version contains supplementary material available at https://doi.org/ 10.1038/s41598-021-91187-2.

Correspondence and requests for materials should be addressed to A.S.-R. or J.A.-B.

Reprints and permissions information is available at www.nature.com/reprints.

Publisher's note Springer Nature remains neutral with regard to jurisdictional claims in published maps and institutional affiliations.

Open Access This article is licensed under a Creative Commons Attribution 4.0 International License, which permits use, sharing, adaptation, distribution and reproduction in any medium or format, as long as you give appropriate credit to the original author(s) and the source, provide a link to the Creative Commons licence, and indicate if changes were made. The images or other third party material in this article are included in the article's Creative Commons licence, unless indicated otherwise in a credit line to the material. If material is not included in the article's Creative Commons licence and your intended use is not permitted by statutory regulation or exceeds the permitted use, you will need to obtain permission directly from the copyright holder. To view a copy of this licence, visit http://creativecommons.org/licenses/by/4.0/.

(C) The Author(s) 2021 